\title{
Téoros
}

Revue de recherche en tourisme

\section{La qualité en tourisme}

La fin justifie les moyens !

\section{Isabelle Proulx et Nathalie Gilbert}

Volume 23, numéro 2, été 2004

La qualité en tourisme

URI : https://id.erudit.org/iderudit/1071299ar

DOI : https://doi.org/10.7202/1071299ar

Aller au sommaire du numéro

Éditeur(s)

Université du Québec à Montréal

ISSN

0712-8657 (imprimé)

1923-2705 (numérique)

Découvrir la revue

Citer ce document

Proulx, I. \& Gilbert, N. (2004). La qualité en tourisme : la fin justifie les moyens !

Téoros, 23(2), 3-4. https://doi.org/10.7202/1071299ar d'utilisation que vous pouvez consulter en ligne.

https://apropos.erudit.org/fr/usagers/politique-dutilisation/ 


\section{Présentation}

\section{La qualité en tourisme La fin justifie les moyens!}

\section{Isabelle Proulx et Nathalie Gilbert}

$\mathbf{L}^{\prime}$ importance du tourisme, sur l'échiquier économique mondial, n'est plus à démontrer. Paradoxalement, le souci d'assurer à cette industrie un développement durable à travers une démarche de contrôle de la qualité s'avère plutôt timide. La préoccupation de voir converger les dimensions économiques, sociales, culturelles et environnementales vers un tourisme durable et de qualité se précise en amont de l'industrie touristique. Prenons pour exemples les positions prises par certaines instances publiques comme l'Organisation mondiale du tourisme avec son Code mondial d'éthique du tourisme ou la récente mise en place d'une politique qualité au Québec. En aval, les entreprises touristiques, bien que certaines marquent des avancées, se trouvent à la croisée du chemin qui mène au virage incontournable de la qualité.

En effet, le contexte de mondialisation amène aujourd'hui les gestionnaires au premier chef, les employés, les fournisseurs, les formateurs et les consommateurs à se préoccuper, sous différents aspects, de qualité. Autant d'intervenants, autant de perceptions de ce qui engendre la qualité. Certains la conçoivent de façon normative, d'autres prônent la déstandardisation. Plusieurs la confinent aux prestations haut de gamme ou l'en dissocient. Quelques-uns la voient difficile à encadrer à travers la multiplicité des contacts humains que comporte l'industrie des services, tandis que d'autres y discernent plutôt une richesse incompa- rable. Trop souvent galvaudé et discuté à travers son aspect commercial, il s'agit d'un sujet que ce dossier de la revue Téoros a voulu décloisonner en cédant la parole à des collaborateurs variés qui ont accepté de livrer leur représentation de la qualité.

Jasmin Tanguay dresse la table et situe l'évolution des attentes des consommateurs en termes de qualité en projetant les changements potentiels au regard des trente dernières années. L'approche normative, introduite dans le monde industriel, servait d'abord l'entreprise avant de servir la clientèle. Aujourd'hui le client, avec ses valeurs postmodernes, recherche un service et un produit personnalisé et non-standardisé. Dans ce contexte, l'employé, formé à servir dans des situations atypiques, devient un important pilier. Dans cette nouvelle phase de développement du concept de qualité, l'auteur explore les avenues et les défis qui se présentent aux acteurs de l'industrie.

La qualité : au cœur de la problématique du tourisme durable ? C'est ce que Saida Merasli explore dans son article qui nous amène à positionner la qualité comme vecteur d'un tourisme durable et responsable. La prise de conscience de cette corrélation est d'abord nécessaire dans l'industrie. L'auteure présente l'évolution de la demande vers un modèle individualisé et l'abondance des outils développés qui font foi de la qualité. Sa réflexion porte sur les difficultés relatives à la fiabilité des outils et suggère la mise en place de critères mesurables et reconnus pour assurer la qualité dans un contexte viable.
Gérard Beaudet poursuit la réflexion dans son article en mettant en relief les bouleversements environnementaux, économiques et socioculturels des milieux d'accueil, malgré le virage qualité effectué au tournant des années 1970 et 1980 ; bouleversements qui auront conduit à une remise en question repositionnée vers le développement d'un tourisme durable qui prend maintenant de nouvelles formes, comme le tourisme vert ou l'écotourisme. Avec la mondialisation économique néolibérale qui amène l'assimilation des identités culturelles, l'industrie touristique se doit d'élargir les initiatives qui s'inscrivent dans une éthique de développement d'un territoire qui dépasse le souci économique.

À travers la perspective marketing de la qualité, y a-t-il confusion entre l'objectif et le moyen ? C'est ce que suggère l'article d'Isabelle Proulx et Nathalie Gilbert qui proposent une vision de la qualité décentrée du client et axée sur l'employé. L'industrie touristique prenant toute sa valeur dans ses multiples interactions humaines, le succès commercial passe davantage par l'orientation employé que l'orientation client. Plutôt que veiller à normaliser l'acte de service, les auteures suggèrent de miser sur une main-d'œuvre formée qui induit l'adaptation d'un comportement souhaitable en tourisme.

Benoît Paquin et Normand Turgeon présentent la qualité par le biais de la matérialisation de l'expérience touristique du consommateur, indicateur perceptible de la qualité par ce dernier. Une stratégie marketing basée sur les aspects matériels «tangibles» du produit touristique l'apparence du personnel en contact ou les 
installations physiques - entraîne la satisfaction de la clientèle. En assurant aux clients une satisfaction au-delà des attentes, les gestionnaires profitent du succès commercial qui résulte du bouche-à-oreille tout en fidélisant la clientèle.

Dans son article Qualité et classification : des enjeux d'importance, Normand Cazelais pose un regard critique sur les limites et l'hétérogénéité des critères de classification, donc de fiabilité. À partir d'une distinction entre les classifications dite officielles, orchestrées par l'État ou l'un de ses mandataires, ou officieuses, déterminées par les entreprises elles-mêmes, l'auteur s'interroge sur la référence objective qu'elles fournissent et la nécessité marketing avouée.

Soucieux de la qualité des infrastructures d'hébergement au Québec, Michel Rheault explique la démarche permettant de respecter la nouvelle Loi sur les établissements d'hébergement touristique qui concerne la classification obligatoire de neuf catégories d'établissement. Son article fournit un aperçu des outils mis en place par la Corporation de l'industrie touristique du Québec (CITQ) pour fournir une information fiable et assurer la protection et la satisfaction du public voyageur.

Danièle Marchand dresse un bilan, le premier, en somme, depuis la mise en place du programme du gouvernement du Québec pour l'amélioration continue de la qualité en tourisme. La lecture de ce constat permet de connaître les tenants et aboutissants de ce projet élaboré et mis en œuvre par Tourisme Québec et ses partenaires. Loin d'être terminée, la réalisation d'une démarche qualité, et surtout ses résultats, demandent un travail continu et de longue haleine. L'auteure termine avec les problématiques auxquelles font face les gestionnaires touristiques impliqués dans le grand projet qui mène à ce virage qualité.

Le sujet n'étant pas épuisé, la collaboration appréciée de Myrabelle Chicoine a permis de rassembler une série de ressources électroniques utiles à la poursuite de nos réflexions. Sans se vouloir exhaustive, la médiagra-

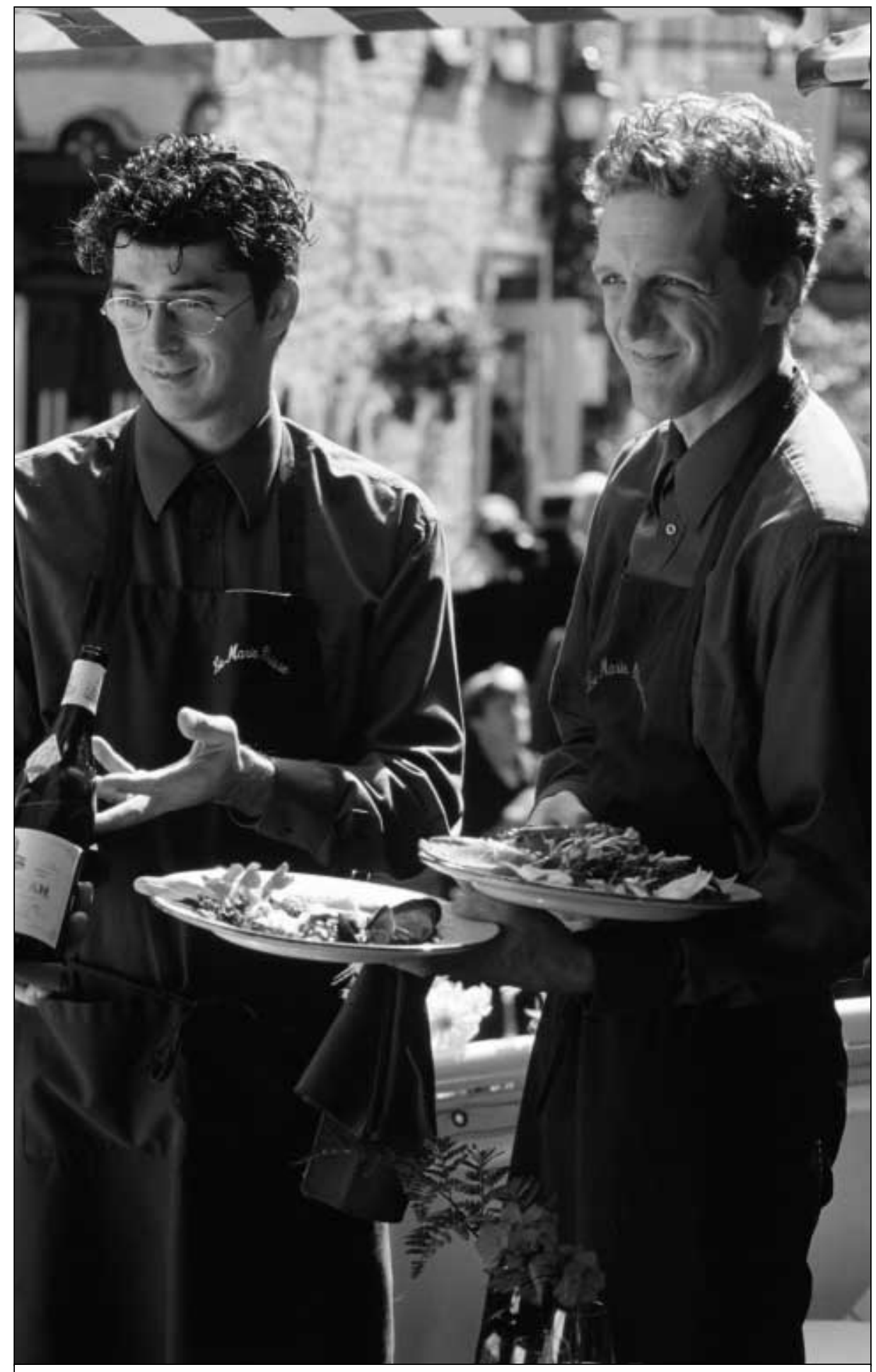

Terrasse Le Marie-Clarisse, Quartier Petit-Champlain, Vieux-Québec. Photo : Tourisme Québec / Linda Turgeon

phie présente des sites qui portent sur la qualité en résumant l'essentiel de leur contenu.

Nous espérons ce numéro de qualité... et nous vous souhaitons bonne lecture!

\section{Isabelle Proulx et Nathalie Gilbert} sont professeures en Techniques de tourisme à l'Institut de tourisme et d'hôtellerie du Québec et sont rédactrices invitées pour ce dossier. 